\title{
PREVALENCIA DEL SÍNDROME DE BURNOUT EN MÉDICOS Y ENFERMERAS DEL PERÚ, ENSUSALUD 2014
}

\author{
Jesús Maticorena-Quevedo ${ }^{1,2, a}$, Renato Beas ${ }^{1,2, a}$, Alexander Anduaga-Beramendi ${ }^{1,2, a}$, \\ Percy Mayta-Tristán ${ }^{1, b}$
}

\begin{abstract}
RESUMEN
Objetivos. Estimar la prevalencia del síndrome de burnout (SB) en los médicos y enfermeras del Perú en el año 2014, según los diferentes puntos de corte establecidos en la literatura. Materiales y métodos. Estudio transversal y descriptivo basado en la Encuesta Nacional de Satisfacción de Usuarios en Salud del año 2014 (ENSUSALUD-2014) que cuenta con un muestreo probabilístico bietápico. EI SB fue identificado mediante el Maslach Burnout Inventory -Human Services Survey (MBI-HSS) utilizando diferentes puntos de corte para establecer su prevalencia: valores predeterminados, terciles y cuartiles. Resultados. De los 5062 profesionales de salud, $62,3 \%$ eran mujeres, $44,0 \%$ eran médicos, $46,0 \%$ pertenecían al MINSA y $23,1 \%$ laboraban en Lima. Se obtuvo una prevalencia global del SB de 2,8\% (IC95\%: 2,19-3,45) usando valores predeterminados; 7,9\% (IC95\%: 6,93-8,95) para puntos de corte según cuartiles; y 12,5\% (IC95\%:11,29-13,77) usando terciles. La prevalencia es mayor en médicos que en enfermeras, independientemente del punto de corte usado $(3,7 \%$ vs $2,1 \%$ en valores predeterminados; 10,2 vs $6,1 \%$ con cuartiles, y 16,2 vs $9,5 \%$ mediante terciles). Conclusiones. La prevalencia del síndrome en personal sanitario es distinta en una misma población, según se utilicen los distintos puntos de corte descritos. Se recomienda el uso de los valores predeterminados por el creador del instrumento, hasta obtener puntos específicos para nuestro país.
\end{abstract}

Palabras clave: Trastornos de Ansiedad; Agotamiento Profesional; Personal de Salud; Prevalencia (fuente: DeCS BIREME).

\section{PREVALENCE OF BURNOUT SYNDROME IN PERUVIAN PHYSICIANS AND NURSES, ENSUSALUD 2014}

\begin{abstract}
Objectives. To estimate the prevalence of burnout syndrome (BOS) in Peruvian physicians and nurses in 2014 according to different cutoff points established in the literature. Materials and methods. This was a cross-sectional and descriptive study based on the National Survey on User Satisfaction of Health Services for 2014 (ENSUSALUD-2014), which features two-stage probability sampling. BOS was identified by the Maslach Burnout Inventory-Human Services Survey (MBI-HSS) using different cutoff points to establish prevalence, including default values, terciles, and quartiles. Results. Of the 5062 health professionals, $62.3 \%$ were women, $44.0 \%$ were physicians, $46.0 \%$ belonged to the MINSA, and $23.1 \%$ worked in Lima. The overall BOS prevalence was $2.8 \%(95 \% \mathrm{Cl}, 2.4-3.2)$, when default values were used; the prevalence was $7.9 \%(95 \% \mathrm{Cl}, 7.3-8.6)$ when quartiles were used as cutoff points and $12.5 \%(95 \% \mathrm{Cl}, 11.4-13.6)$ when terciles were used as cutoff points. The prevalence was higher in doctors than in nurses, regardless of the cutoff point used $(3.7 \%$ vs. $2.1 \%$ using default values, 10.2 vs. $6.1 \%$ using quartiles, and 16.2 vs. $9.5 \%$ using terciles). Conclusions. The prevalence of BOS in health workers differs within the same population when different cutoff points are used. The use of default values is recommended by the instrument author until specific cut-points for our country are obtained.
\end{abstract}

Key words: Anxiety Disorders; Burnout, Professional; Health Personnel; Prevalence (source: MeSH NLM).

\section{INTRODUCCIÓN}

El síndrome de burnout (SB) fue identificado por primera vez por Maslach y Jackson en los años 70, y es considerado un estado de estrés psicológico y emocional prolongado en el trabajo, caracterizado por tres dimensiones clave: agotamiento emocional, despersonalización, y sentido reducido de la realización personal ${ }^{(1)}$. Este síndrome fue inicialmente identificado en profesionales cuya actividad laboral se fundamenta en la interacción con personas como enfermeras, médicos, profesores, etc. Sin embargo, actualmente es considerada una condición que puede afectar a cualquier individuo (2). En los profesionales de la salud,

\footnotetext{
Escuela de Medicina, Universidad Peruana de Ciencias Aplicadas. Lima, Perú

Sociedad Científica de Estudiantes de Medicina de la Universidad Peruana de Ciencias Aplicadas (SOCIEMUPC). Lima, Perú

Estudiante de medicina; ${ }^{\mathrm{b}}$ médico salubrista

Recibido: 12/01/2016 Aprobado: 20/04/2016
}

Citar como: Maticorena-Quevedo J, Beas R, Anduaga-Beramendi A, Mayta-Tristán P. Prevalencia del síndrome de burnout en médicos y enfermeras del Perú, Ensusalud 2014. Rev Peru Med Exp Salud Publica. 2016;33(2):241-7. doi: 10.17843/rpmesp.2016.332.2170 
el SB ha sido ampliamente estudiado y se ha relacionado a sobrecarga laboral, deseo de cambiar de trabajo e incluso, en algunos países, el SB es considerado un diagnóstico médico-legal tributario de descanso médico ${ }^{(2)}$.

Para identificar sujetos con SB se han propuesto diversos instrumentos dependiendo de la población; sin embargo, el Maslach Burnout Inventory (MBI) es considerado el gold standard, debido a que es consistente, psicométricamente adecuado, fácil de usar y el más usado a nivel mundial ${ }^{(2)}$. Para la evaluación del SB en profesionales de la salud se utiliza la versión MBI-HSS (Maslach Burnout Inventory - Human Services Survey), adaptada al personal sanitario, donde se considera como caso positivo de SB al individuo que tenga puntuaciones altas en las dimensiones de $A E$ y DP además de puntajes bajos en RP ${ }^{(1,2)}$.

Para la clasificación de los puntajes en altos, medios y bajos en cada subescala, se han usado distintos puntos de corte ${ }^{(1,3,4)}$. Existen valores predeterminados propuestos por Maslach et al. para cualquier población, habiéndose obtenido estos valores arbitrariamente mediante terciles a partir de población norteamericana ${ }^{(1)}$. Siguiendo esa premisa, otros autores han reproducido esta modalidad de categorización en terciles para sus poblaciones, obteniendo sus propios puntos de corte $\mathrm{e}^{(1,4,5)}$ Por otro lado, existe otra forma de interpretación de este instrumento utilizando límites más alejados de la media, como son los cuartiles ${ }^{(4,6)}$. De esta manera, en la literatura existen diversas formas de cuantificar los valores de cada dimensión del MBI-HSS.

Muchos autores incluidos los creadores del instrumento, consideran que este síndrome debe ser analizado por cada dimensión de manera independiente en forma numérica y valorar la asociación de cada una con diversos factores relacionados al trabajo ${ }^{(2,5)}$. Sin embargo, desde el punto de vista de salud pública, otros autores afirman que este tipo de análisis no contribuye a tomar medidas en la población afectada ya que no establece prevalencias y, por ello, se debe categorizar el SB en caso positivo o negativo ${ }^{(7,8)}$. En este sentido, la operacionalización y medición del SB como una variable dicotómica ha tenido múltiples interpretaciones, teniendo puntos de corte distintos de acuerdo al modelo usado y obteniéndose prevalencias muy variables entre 4 y $66 \%$ en el personal de salud ${ }^{(5,9)}$.

En el Perú, desde el año 2014, la Superintendencia Nacional de Salud (SUSALUD) mide el SB mediante el MBI-HSS como parte de la evaluación de satisfacción del usuario interno de los servicios de salud ${ }^{(10)}$. No obstante, al no existir un consenso acerca de los puntos de corte que utilizar, no se puede identificar con certeza la prevalencia del SB en dicha población, en consecuencia, se podría sobreestimar o disminuir el real impacto del síndrome. Por esta razón, el objetivo del estudio fue estimar la prevalencia de síndrome de burnout en los médicos y enfermeras de Perú en el año 2014 , según los diferentes puntos de corte establecidos, y compararlo con otros estudios.

\section{MATERIALES Y MÉTODOS}

Estudio de corte transversal descriptivo. Se llevó a cabo un análisis secundario de datos de la Encuesta Nacional de Satisfacción de Usuarios de Salud del año 2014 (ENSUSALUD-2014), realizada por SUSALUD y el Instituto Nacional de Estadística e Informática (INEI) con fines de valorar la satisfacción del usuario interno y externo. Para el presente artículo se consideró el cuestionario para personal médico y de enfermería ${ }^{(10)}$

El muestreo fue probabilístico, estratificado e independiente en cada uno de los establecimientos de salud pertenecientes al Ministerio de Salud (MINSA), Seguro Social (EsSalud), clínicas privadas y sanidad de las fuerzas armadas y policiales, de los 24 departamentos del Perú. La muestra final se obtuvo en dos etapas: en la primera, mediante selección aleatoria simple de cada establecimiento según la categoría proporcionada por SUSALUD, y en la segunda, mediante selección de profesionales de salud, médicos y enfermeras, de manera sistemática, de acuerdo al número de atenciones por establecimiento ${ }^{(10)}$.

El tamaño muestral fue de 5067 profesionales de salud, calculado para estimar a nivel nacional y regional la prevalencia de insatisfacción del usuario interno en las instituciones de salud, considerando una insatisfacción del $40 \%$, nivel de confianza del $95 \%$ y un margen de error entre 3 a 9 puntos porcentuales por región. Dado que los márgenes de error son potencialmente más grandes que prevalencias halladas de $\mathrm{SB}$, solo se calculará las prevalencias nacionales y no regionales.

La ejecución del trabajo de campo estuvo a cargo de la Dirección Nacional de Censos y Encuestas (DNCE) y se realizó del 24 de marzo al 7 de mayo del 2014 . Las encuestas fueron heteroaplicadas por personal previamente capacitado. Hubo 5,9\% de entrevistas cuyo resultado final fue: rechazo rotundo, rechazo por falta de tiempo u otros. Para el presente estudio se excluyó a cinco profesionales, debido a que no completaron algunas de las preguntas del MBI-HSS, resultando una muestra final de 5062 profesionales de salud.

El síndrome de burnout se midió usando el MBI-HSS, instrumento modificado en 1986 y validado al idioma español para el personal de salud, el cual consta de 22 ítems con escala Likert del 0 al 6 y con un alfa de Cronbach global de $0,86^{(1,7)}$. A nuestro conocimiento este instrumento no cuenta con una validación para la 
población peruana. EI MBI-HSS mide tres dimensiones: agotamiento emocional (AE); despersonalización (DP), y realización personal (RP) (1): a) El AE consta de 9 ítems, con un valor máximo de 54 y un alfa de Cronbach del instrumento validado al español de 0,85. b) La DP posee de 5 ítems, su valor máximo es de 30 y tiene un alfa de Cronbach de 0,58. c) La RP está compuesta por 8 componentes, su valor máximo es de 48 y tiene un alfa de Cronbach de $0,71^{(3,7)}$. Las características de cada dimensión del MBI-HSS se observan en la Tabla 1.

Se definió como caso de burnout al sujeto que presentara valores altos en las dimensiones de AE y DP y bajos en $\mathrm{RP}$, según tres modelos de interpretación encontrados en la literatura: a). Valores predeterminados: $A E>26$, $\mathrm{DP}>9 \mathrm{RP}<34^{(1,3)}$; b). Según clasificación por cuartiles de cada subescala, teniendo como punto de corte los percentiles 75 y 25 para alto o bajo, respectivamente ${ }^{(4,6)}$, y c). Según clasificación por terciles de cada subescala, donde un valor alto de cada dimensión se encuentra por encima del percentil 66 , y bajo, por debajo de $33^{(1,5)}$

Se evaluaron variables sociodemográficas como edad (terciles); sexo (varón, mujer); estado civil (casado, soltero, conviviente, otros); especialidad (sí, no); residencia en Lima metropolitana (sí, no); entidad para la que trabaja (MINSA, EsSalud, FF.AA., clínicas privadas); categoría del establecimiento para el que trabaja según MINSA (I o primer nivel de atención, II o nivel medio y III o nivel de mayor complejidad) e ingresos mensuales. Para el análisis de las variables categóricas se utilizó frecuencias y porcentajes. Se categorizó las variables numéricas de acuerdo a terciles.

Parala ejecución de la encuesta se realizó un consentimiento informado verbal por parte de los evaluadores técnicos de la encuesta, previa capacitación (10). Los datos de ENSUSALUD 2014 provienen de una base de datos pública, disponible en forma anónima en la web del INEI.

El análisis estadístico se realizó con el software estadístico STATA 14.0. Se calcularon las prevalencias ponderadas usando el comando svy y se estimó el número total de profesionales con burnout considerando los factores de expansión.

\section{RESULTADOS}

Las dimensiones del SB mostraron una adecuada consistencia interna para la población analizada, obteniéndose valores de alfa de Cronbach de 0,84 para $\mathrm{AE} ; 0,71$ para DP y 0,75 para RP como se ve en la Tabla 1 .

De la población estudiada, el $62,3 \%$ fueron mujeres, $44,0 \%$ eran médicos, $46,0 \%$ pertenecían al MINSA y $23,1 \%$ laboraban en Lima. El promedio de edad de los participantes fue de $44,2( \pm 10,9)$. El promedio de años de trabajo en el sector salud fue $16,5( \pm 10.1)$ con un mínimo de 1 y un máximo de 57 . Las características sociodemográficas de la población se observan en la tabla 2.

La prevalencia del SB fue mayor en médicos con respecto a las enfermeras, independientemente del punto de corte usado $(3,7$ vs $2,1 \%$ en valores predeterminados; 10,2 vs $6,1 \%$ con cuartiles; y 16,2 vs $9,5 \%$ usando terciles como puntos de corte). La dimensión del MBIHSS con mayor prevalencia en ambas poblaciones fue la baja RP: 19,4\% (IC95\%: 17,91-20,80) con valores predeterminados, 37,7\% (IC95\%:35,88-39,46) para terciles y 26,4\% (IC95\%:24,74-27,97) según el análisis por cuartiles. Asimismo, la baja RP fue la única subescala del MBI-HSS que estuvo más presente en enfermeras en los 3 análisis $(20,4 \%, 26,9 \%$ y $38,1 \%$, respectivamente).

Se obtuvo una prevalencia global del SB de 2,8\% (IC95\%: 2,19-3,45) usando valores predeterminados; 7,9\% (IC95\%: 6,93-8,95) para puntos de corte según cuartiles; y 12,5\% (IC95\%:11,29-13,77) usando terciles según lo muestra la tabla 3 . Se estimó que el número total de trabajadores de salud que padecen de SB en el Perú, siguiendo los puntos de corte de Maslach et al., sería de 1182 (IC95\%: 916 - 1448) profesionales.

Tabla 1. Características de las dimensiones del MBI-HSS

\begin{tabular}{lccr}
\hline Características & Agotamiento emocional & Despersonalización & Realización personal \\
\hline Número de ítems & 9 & 5 & 8 \\
Puntaje (min - máx) & $0-54$ & $0-30$ & $0-48$ \\
Alfa de Cronbach* & 0,84 & 0,71 & 0,75 \\
Mediana (p25-p75) & $10(5-17)$ & $3(1-6)$ & $40(35-43)$ \\
Media (DE) & $12,0(9,3)$ & $4,2(4,5)$ & $38,3(7,7)$ \\
Puntos de corte & & & \\
VP & $>26$ & $>9$ & $<34$ \\
Terciles & $>15$ & $>6$ & $<38$ \\
Cuartiles & $>18$ & $>7$ & $<35$ \\
\hline
\end{tabular}

VP: valores predeterminados, DE: desviación estándar

* Valores obtenidos en la población evaluada 
Tabla 2. Características de la población entre el personal sanitario en Perú, ENSUSALUD 2014

\begin{tabular}{|c|c|c|c|c|c|c|}
\hline \multirow{2}{*}{ Características } & \multicolumn{2}{|c|}{ Global } & \multicolumn{2}{|c|}{ Médicos } & \multicolumn{2}{|c|}{ Enfermeras } \\
\hline & $n=5062$ & $(\%)$ & $n=2228$ & $(\%)$ & $\mathrm{n}=\mathbf{2 8 3 4}$ & $(\%)$ \\
\hline \multicolumn{7}{|l|}{ Sexo } \\
\hline Varón & 1907 & $(37,7)$ & 1697 & $(76,2)$ & 210 & $(7,4)$ \\
\hline Mujer & 3155 & $(62,3)$ & 531 & $(23,8)$ & 2624 & $(92,6)$ \\
\hline \multicolumn{7}{|l|}{ Estado civil } \\
\hline Casado & 2903 & $(57,3)$ & 1421 & $(63,8)$ & 1482 & $(52,3)$ \\
\hline Soltero & 1332 & $(26,3)$ & 512 & $(23,0)$ & 820 & $(28,9)$ \\
\hline Conviviente & 452 & $(8,9)$ & 148 & $(6,6)$ & 304 & $(10,7)$ \\
\hline Otros & 375 & $(7,4)$ & 147 & $(6,6)$ & 228 & $(8,0)$ \\
\hline \multicolumn{7}{|l|}{ Edad } \\
\hline <39 años & 1795 & $(35,5)$ & 718 & $(32,2)$ & 1077 & $(38,0)$ \\
\hline 39-50 años & 1698 & $(33,5)$ & 756 & $(33,9)$ & 942 & $(33,2)$ \\
\hline >50 años & 1569 & $(31,0)$ & 754 & $(33,8)$ & 815 & $(28,8)$ \\
\hline \multicolumn{7}{|l|}{ Especialidad } \\
\hline Sí & 2917 & $(57,6)$ & 1472 & $(66,1)$ & 1445 & $(51,0)$ \\
\hline No & 2145 & $(42,4)$ & 756 & $(33,9)$ & 1389 & $(49,0)$ \\
\hline \multicolumn{7}{|l|}{ Ingresos (soles) } \\
\hline$<2000$ & 994 & $(19,6)$ & 95 & $(4,3)$ & 899 & $(31,7)$ \\
\hline $2000-3000$ & 1571 & $(31,0)$ & 163 & $(7,3)$ & 1408 & $(49,7)$ \\
\hline $3000-5000$ & 1715 & $(33,9)$ & 1213 & $(54,4)$ & 502 & $(17,7)$ \\
\hline$>5000$ & 782 & $(15,5)$ & 757 & $(34,0)$ & 25 & $(0,9)$ \\
\hline \multicolumn{7}{|l|}{ Lima Metropolitana } \\
\hline Sí & 1168 & $(23,1)$ & 540 & $(24,2)$ & 628 & $(22,2)$ \\
\hline No & 3894 & $(76,9)$ & 1688 & $(75,8)$ & 2,206 & $(77,8)$ \\
\hline \multicolumn{7}{|l|}{ Institución } \\
\hline MINSA & 2329 & $(46,0)$ & 1009 & $(45,3)$ & 1320 & $(46,6)$ \\
\hline EsSalud* & 2318 & $(45,8)$ & 1044 & $(46,9)$ & 1274 & $(45,0)$ \\
\hline Sanidades $†$ & 97 & $(1,9)$ & 36 & $(1,6)$ & 61 & $(2,2)$ \\
\hline Privadas & 318 & $(6,3)$ & 139 & $(6,2)$ & 179 & $(6,3)$ \\
\hline \multicolumn{7}{|l|}{ Categoría } \\
\hline I (Primer nivel) & 553 & $(10,9)$ & 248 & $(11,1)$ & 305 & $(10,8)$ \\
\hline II (Segundo nivel) & 2630 & $(52,0)$ & 1128 & $(50,6)$ & 1502 & $(53,0)$ \\
\hline III (Mayor complejidad) & 1879 & $(37,1)$ & 852 & $(38,2)$ & 1027 & $(36,2)$ \\
\hline
\end{tabular}

* Seguro Social † Fuerzas Armadas y Policía Nacional del Perú

\section{DISCUSIÓN}

Los resultados presentados muestran diferencia según los puntos de corte utilizados para el diagnóstico del SB. En el análisis mediante terciles se obtuvo la mayor prevalencia, seguido por el de cuartiles y por el de valores predeterminados. Estos resultados revelan que, según el modo de interpretación de los puntos de corte, la diferencia en la prevalencia de SB puede ser de hasta cinco veces, lo que podría sobreestimar o subestimar la ocurrencia de este síndrome. Debido a que SUSALUD ha establecido la medición del SB en los profesionales de la salud de manera periódica en el país, esta diferencia de prevalencias podría repercutir en la interpretación del estado actual de este síndrome en esa población y en las medidas de salud pública que se tomen.
Es necesario mencionar que el uso de terciles y cuartiles como puntos de corte es útil para identificar los factores asociados al SB. Sin embargo, las prevalencias del SB serían muy dependientes del total de muestra analizada y de la distribución de datos de la misma. También, en caso se lleven a cabo estrategias para disminuir el SB, no se podría valorar el impacto de estas intervenciones en el personal de salud si se usara este tipo de análisis. Este obstáculo de no poder comparar los resultados de las intervenciones en una misma población limita el uso de la interpretación mediante terciles y cuartiles. Por otra parte, el uso de valores predeterminados permite determinar prevalencias diferentes según la muestra y así se puede observar el efecto de las acciones tomadas por un sistema de salud o entidad investigadora ${ }^{(11)}$. Si bien existe puntos de corte establecidos para ciertos 
países y por los creadores del instrumento, en el Perú no existen estudios donde se haya establecido valores de corte específicos para nuestra población ${ }^{(1,8,12)}$.

Se ha afirmado que la validez transcultural de los valores predeterminados obtenidos por Maslach et al. a partir de población estadounidense es cuestionable; y que los puntos de corte deberían ser específicos para cada país para responder a características locales, roles tradicionales de sexo y otros factores; sin embargo, estos valores predeterminados han sido ampliamente utilizados alrededor del mundo para medir el SB y permiten estandarizar el análisis de este síndrome para su comparación en diferentes poblaciones o en una misma población en distintos momentos; por lo que su uso en la población peruana debe ser recomendado ${ }^{(11,13)}$. Por esta razón, se realizó una búsqueda de artículos que mencionen el uso del MBI-HSS para obtener la prevalencia del SB en diferentes países, según los valores predeterminados, la cual se muestra en la Tabla 4. Se excluyeron los artículos con poblaciones menores a 50 personas.

De los artículos encontrados en la búsqueda, el presente estudio es el que tiene la muestra más amplia. Solo se encontraron dos estudios probabilísticos realizados por Navarro-González y Parada ${ }^{(16,19)}$. El presente estudio tiene una muestra representativa del personal de salud de todo el país, sin discriminación de especialidad. Asimismo, se puede identificar prevalencias de SB de hasta $66 \%$ en profesionales de salud de especialidades específicas, como pediatría, cirugía y anestesiología cardiovascular, emergencia, etc. ${ }^{(18,25,26)}$. Esto quizás se deba a que se incluyó poblaciones más susceptibles al SB.

Con respecto a la prevalencia encontrada mediante valores predeterminados, el valor a nivel de todo el personal de salud fue el menor de todos, incluso inferior al encontrado por Vilá Falguera et al. quienes utilizaron los mismos puntos de corte, y donde se obtuvo una prevalencia de $3,4 \%$; esto se podría explicar por el distinto ambiente laboral y la inclusión de solo el personal de salud que laboraba en el sector público ${ }^{(14)}$. Por otra parte, la prevalencia de SB por puntos de corte mediante terciles en el presente estudio fue de $12,5 \%$, muy parecida a la encontrada por Grau et al. en población ibérica e hispanoamericana bajo esa misma modalidad de análisis ${ }^{(11)}$.

Otro aspecto interesante del estudio radica en la diferencia entre los puntos de corte utilizados para esta población en cada dimensión del SB, utilizando terciles y cuartiles, y los valores predeterminados por los creadores del instrumento, como se aprecia en la Tabla 1. Si bien, existe semejanza entre los puntos de corte para valores altos en las dimensiones de RP y DP bajo las tres formas de análisis; los valores altos para la dimensión de $\mathrm{AE}$ tienen un punto de corte mayor en los valores predeterminados difiriendo este valor hasta en 11 unidades comparado con los puntos de corte mediante terciles y cuartiles.

En este sentido, se refuerza la idea de Schaufeli et al. respecto de la influencia de las características locales de la población para la determinación del SB (13). Por consiguiente, esta gran brecha en dicha dimensión contribuye a que la prevalencia global del SB utilizando valores predeterminados sea la más baja de todas.

De la misma forma, se observa en el estudio que, independientemente de los puntos de corte utilizados, la prevalencia de SB es mayor en médicos en comparación con las enfermeras. Estos hallazgos coinciden con los de Grau et al. y esbozan que probablemente el personal médico se encuentra más expuesto a factores que influyen en el burnout y el $\mathrm{AE}$ como son: mayor

Tabla 3. Prevalencia de síndrome de burnout en el personal sanitario peruano según puntos de corte, ENSUSALUD 2014

\begin{tabular}{|c|c|c|c|c|c|c|}
\hline \multirow{2}{*}{ Grupos } & \multicolumn{2}{|c|}{ Valores predeterminados } & \multicolumn{2}{|c|}{ Terciles } & \multicolumn{2}{|c|}{ Cuartiles } \\
\hline & Prevalencia & (IC95\%) & Prevalencia & (IC95\%) & Prevalencia & (IC95\%) \\
\hline \multicolumn{7}{|l|}{ Total } \\
\hline Global & 2,8 & $(2,19-3,45)$ & 12,5 & $(11,29-13,77)$ & 7,9 & $(6,93-8,95)$ \\
\hline DP & 12,8 & $(11,58-14,05)$ & 30,4 & $(28,69-32,09)$ & 24,5 & $(22,95-26,13)$ \\
\hline $\mathrm{AE}$ & 10,6 & $(9,46-11,81)$ & 34,0 & $(32,23-35,78)$ & 25,5 & $(23,91-27,17)$ \\
\hline $\mathrm{RP}$ & 19,4 & $(17,91-20,80)$ & 37,7 & $(35,88-39,46)$ & 26,4 & $(24,74-27,97)$ \\
\hline \multicolumn{7}{|l|}{ Médicos } \\
\hline Global & 3,7 & $(2,66-4,82)$ & 16,2 & $(14,15-18,31)$ & 10,2 & $(8,52-11,89)$ \\
\hline DP & 16,8 & $(14,69-18,87)$ & 36,7 & $(33,97-39,32)$ & 29,7 & $(27,16-32,24)$ \\
\hline $\mathrm{AE}$ & 14,2 & $(12,20-16,16)$ & 40,3 & $(37,53-43,01)$ & 31,8 & $(29,16-34,36)$ \\
\hline $\mathrm{RP}$ & 18,1 & $(16,00-20,20)$ & 37,1 & $(34,47-39,81)$ & 25,7 & $(23,28-28,08)$ \\
\hline \multicolumn{7}{|c|}{ Enfermeras } \\
\hline Global & 2,1 & $(1,34-2,80)$ & 9,5 & $(8,03-10,94)$ & 6,1 & $(4,88-7,27)$ \\
\hline DP & 9,6 & $(8,13-10,98)$ & 25,2 & $(23,08-27,37)$ & 20,3 & $(18,30-22,27)$ \\
\hline $\mathrm{AE}$ & 7,7 & $(6,34-9,09)$ & 28,8 & $(26,56-31,10)$ & 20,4 & $(18,39-22,45)$ \\
\hline $\mathrm{RP}$ & 20,4 & $(18,40-22,39)$ & 38,1 & $(35,69-40,51)$ & 26,9 & $(24,72-29,10)$ \\
\hline
\end{tabular}

DP: despersonalización, AE: agotamiento emocional, RP: realización personal 
Tabla 4. Prevalencia del síndrome de burnout según valores predeterminados en el personal de salud

\begin{tabular}{|c|c|c|c|c|c|}
\hline Autor & País, año & Lugar(n) & Especialidad & Muestra, muestreo & $(\%)$ \\
\hline \multicolumn{6}{|l|}{ Todo personal } \\
\hline Maticorena-Quevedo & Perú, 2014 & H. públicos y privados (181) & General & $5067, \mathrm{P}$ & $(2,8)$ \\
\hline Falgueras MV ${ }^{(14)}$ & España, 2015 & H. públicos & General & $879, \mathrm{C}$ & $(3,4)$ \\
\hline Vásquez-Manrique (15) & Perú, 2014 & H. público (1) & General & $54, \mathrm{C}$ & $(3,8)$ \\
\hline Ortega $^{(3)}$ & Panamá, 2011 & H. público (1) & & $156, C$ & $(4,5)$ \\
\hline Navarro-González ${ }^{(16)}$ & España, 2015 & H. públicos (54) & Atención Primaria & $178, P$ & $(4,5)$ \\
\hline Silva SC ${ }^{(17)}$ & Brasil, 2015 & H. público (1) & Atención Primaria & 194, NP & $(7,0)$ \\
\hline Popa ${ }^{(18)}$ & Romania, 2010 & H. públicos & Emergencia & 4693, NP & $(12,8)$ \\
\hline \multicolumn{6}{|l|}{ Enfermeras } \\
\hline Maticorena-Quevedo & Perú, 2014 & H. públicos y privados (181) & General & $5067, \mathrm{P}$ & $(2,8)$ \\
\hline Grau-Alberola ${ }^{(11)}$ & España, 2010 & H. públicos (13) & General & $316, \mathrm{NP}$ & $(2,8)$ \\
\hline Parada ${ }^{(19)}$ & Venezuela, 2005 & H. universitario (1) & General & $104, P$ & $(6,7)$ \\
\hline Ribeiro ${ }^{(20)}$ & Brasil, 2014 & H. privado (1) & General & $118, \mathrm{NP}$ & $(10,1)$ \\
\hline Xiao-Chun (21) & China, 2014 & H. públicos (10) & Cuidados Intensivos & $413, \mathrm{NP}$ & $(16,0)$ \\
\hline \multicolumn{6}{|l|}{ Médicos } \\
\hline Leung ${ }^{(22)}$ & $\begin{array}{l}\text { Australia, Nueva } \\
\text { Zelanda, } 2015\end{array}$ & SP. radiólogos & Radiología oncológica & $220, C$ & $(3,0)$ \\
\hline Maticorena-Quevedo & Perú, 2014 & H. públicos y privados (181) & General & $5067, \mathrm{P}$ & $(3,7)$ \\
\hline Magalhaes ${ }^{(23)}$ & Brasil, 2015 & SP. anestesiología & Anestesiólogos & $134, \mathrm{NP}$ & $(10,4)$ \\
\hline Gil-Monte ${ }^{(12)}$ & Argentina, 2008 & H. públicos & Pediatría & $123, \mathrm{NP}$ & $(10,6)$ \\
\hline Soler ${ }^{(24)}$ & Europa, 2008 & SP. europea & Atención Primaria & $1393, \mathrm{NP}$ & $(12,0)$ \\
\hline Mikalauskas ${ }^{(25)}$ & Lituania, 2011 & H. universitarios y público (3) & Cirugía y anestesiología & $59, \mathrm{NP}$ & $(62,0)$ \\
\hline Martins ${ }^{(26)}$ & Argentina, 2011 & H. público (1) & Pediatría & $117, \mathrm{NP}$ & $(66,0)$ \\
\hline
\end{tabular}

H: hospital, Es: establecimientos, SP: sociedad profesional

$\mathrm{P}$ : probabilístico, NP: no probabilístico, C: censal

sobrecarga de trabajo, privación del sueño, conflictos laborales y bajo compromiso organizacional $(5,27)$. Además, en nuestro estudio se observó semejanzas solo en la dimensión de RP entre médicos y enfermeras, lo que contrasta con Atance et al., quienes mostraron que los puntajes obtenidos para cada dimensión de SB eran muy semejantes en los dos grupos, y Schaufeli et al. que afirman que los médicos y las enfermeras experimentan un nivel comparable de $A E{ }^{(28,29)}$. Lo anterior, se explicaría por el escaso trabajo interdisciplinario dentro de los establecimientos de salud de nuestro país, lo que podría sobrecargar de labores a ciertos profesionales.

También se encontró una mayor prevalencia de niveles bajos de RP en comparación con las prevalencias de niveles altos de AE y DP, bajo las tres formas de análisis. Estos resultados coinciden con lo encontrado por Caballero et al. en una población madrileña de médicos, enfermeras y auxiliares de salud, donde la prevalencia de valores altos de AE y DP fue inferior a la de valores bajos de RP que alcanzó el 50,3\% ${ }^{(30)}$. Esto se puede explicar por las carencias que aún posee el sistema de salud peruano en cuanto a capacitación, remuneración, reconocimiento del mérito y otros beneficios laborales de los profesionales. El estudio presenta ciertas limitaciones, el no haber considerado la población de médicos y enfermeras que laboran de manera independiente dentro del sistema de salud, podría restar una fracción importante susceptible de SB; asimismo, no se consideraron a otros profesionales de la salud que podrían estar afectados por este síndrome, sin embargo la muestra es bastante representativa. Finalmente, en la actualidad no se ha hecho la adaptación cultural del MBI-HSS al Perú; sin embargo, se encontró una alta consistencia interna.

En conclusión, la prevalencia del síndrome en el personal sanitario es distinta en una misma población estudiada, según se utilicen los distintos puntos de corte descritos.

Además, debido a que es necesario conocer la prevalencia del SB para la implementación de medidas de salud pública, se recomienda el uso de los valores predeterminados por el creador del instrumento hasta obtener puntos específicos para nuestro país.

Contribuciones de autoría: JMQ, RBN, AAB y PMT han participado en la concepción y diseño del artículo, revisión crítica del artículo y aprobación para su versión final. JMQ, RBN y AAB escribieron el borrador del manuscrito y PMT realizó el análisis e interpretación de datos.

Fuente de financiamiento: autofinanciado

Conflictos de interés: los autores declaramos no tener conflictos de interés 


\section{REFERENCIAS BIBLIOGRÁFICAS}

1. Maslach C, Jackson SE, Leiter MP. Maslach Burnout Inventory. $3^{\mathrm{a}}$ ed. The Scarecrow Press; 1996. p. 191-218.

2. Maslach C, Leiter M, Schaufeli W. Measuring burnout. Oxford: The Oxford handbook of organizational well-being; 2008. p. 86-108.

3. Loubon CO, Salas R, Correa R. Aspectos epidemiológicos del síndrome de burnout en el personal sanitario. Hospital aquilino tejeira. Febreromarzo 2011. Archivos de medicina. 2011;7(2):1-7. doi: 10:3823/072

4. Lorenz VR, Benatti MCC, Sabino MO. Burnout y estrés en enfermeros de un hospital universitario de alta complejidad. Rev Latino-Am Enfermagen [internet]. Nov-dec 2010 [citado el 25 de abril de 2015];18(6):[9 pág.]. Disponible en: http://www. scielo.br/pdf/rlae/v18n6/es_07.pdf

5. Grau Martin A, Flichtentrei D, Suner $R$, Prats $M$, Braga F. Influencia de factores personales, profesionales y transnacionales en el síndrome de burnout en personal sanitario hispanoamericano y español (2007). Rev Esp Salud Publica. 2009;83(2):215-30.

6. Lautert L. O desgaste profissional: estudo empírico com enfermeiras que trabalham em hospitais. Rev Gaúch Enferm. 1997;18(2):133-44.

7. Gil-Monte PR. Factorial validity of the Maslach Burnout Inventory (MBIHSS) among Spanish professionals. Rev Saude Publica. 2005;39(1):1-8.

8. Schaufeli WB, Bakker AB, Hoogduin $\mathrm{K}$, Schaap C, Kladler A. On the clinical validity of the Maslach Burnout Inventory and the Burnout Measure. Psychol Health. 2001;16(5):565-82. doi: 10.1080/08870440108405527.

9. Dréano-Hartz $S$, Rhondali W, Ledoux M, Ruer M, Berthiller J, Schott A-M, et al. Burnout among physicians in palliative care: Impact of clinical settings. Palliat Support Care. 2015:19. doi: $10.1017 /$ S1478951515000991

10. Instituto Nacional de Estadística e Informática (INEI). Superintendencia Nacional de Aseguramiento en Salud (SUNASA). Encuesta nacional de satisfacción de usuarios del aseguramiento universal en salud. Lima: INEI/SUNASA; 2014.

11. Grau-Alberola E, Gil-Monte PR, Garcia-Juesas JA, Figueiredo-Ferraz H. Incidence of burnout in Spanish nursing professionals: a longitudinal study. Int J Nurs Stud. 2010;47(8):1013-20. doi: 10.1016/j.ijnurstu.2009.12.022.
12. Gil-Monte PR, Marucco M Prevalencia del "síndrome de quemarse por el trabajo"(burnout) en pediatras de hospitales generales. Rev Saude Publica. 2008;42(3):450-6.

13. Schaufeli WB, Van Dierendonck D. A cautionary note about the cross-national and clinical validity of cut-off points for the Maslach Burnout Inventory. Psychol Rep. 1995;76(3 Pt 2):1083-90.

14. Vilà Falgueras $M$, Cruzate Muñoz C, Orfila Pernas F, Creixell Sureda J, González López MP, Davins Miralles J. Burnout y trabajo en equipo en los profesionales de Atención Primaria. Atención Primaria. 2015;47(1):25-31. doi:10.1016/j.aprim.2014.01.008

15. Vásquez-Manrique JF, Maruy-Saito A, Verne-Martin E. Frecuencia del síndrome de burnout y niveles de sus dimensiones en el personal de salud del servicio de emergencia de pediatría del Hospital Nacional Cayetano Heredia en el año 2014: Lima, Perú. Rev Neuropsiquiatr. 2014;77(3):168-74.

16. Navarro-González D, Ayechu-Diaz A, Huarte-Labiano I. Prevalencia del síndrome del burnout y factores asociados a dicho síndrome en los profesionales sanitarios de Atención Primaria. Semergen. 2014;41(4):191-8. doi: 10.1016/j.semerg.2014.03.008.

17. Silva SC, Nunes MA, Santana VR, Reis FP, Machado Neto J, Lima SO. Burnout syndrome in professionals of the primary healthcare network in Aracaju, Brazil. Cien Saude Colet. 2015;20(10):3011$20 . \quad$ doi: $10.1590 / 1413$ 812320152010.19912014

18. Popa F, Arafat R, Purcarea VL, Lala A, Bobirnac G. Occupational burnout levels in emergency medicine-a nationwide study and analysis. J Med Life. 2010;3(3):207-15.

19. Parada ME, Moreno B, Mejías M, Rivas F, Cerrada S, Rivas P. Satisfacción laboral y síndrome de burnout en el personal de enfermería del Instituto Autónomo Hospital Universitario Los Andes (IAHULA), Mérida, Venezuela, 2005. Rev Fac Nac Salud Publica. 2005;23(1):33-45.

20. Ribeiro VF, Ferreira FC, Valenti VE, Ferreira M, de Abreu LC, de Carvalho $\mathrm{TD}$, et al. Prevalence of burnout syndrome in clinical nurses at a hospital of excellence. Int Arch Med. 2014;7:22. doi: 10.1186/1755-7682-7-22.

21. Zhang XC, Huang DS, Guan P, SUBLIN Study Team. Job burnout among critical care nurses from 14 adult intensive care units in northeastern
China: a cross-sectional survey. BMJ open. 2014;4(6):e004813. doi: 10.1136/bmjopen-2014-004813.

22. Leung J, Rioseco P, Munro P. Stress, satisfaction and burnout amongst Australian and New Zealand radiation oncologists. J Med Imaging Radiat Oncol. 2015;59(1):115-24. doi: 10.1111/1754-9485.12217.

23. Magalhães E, Oliveira ÁC, Govêia CS, Ladeira LC, Queiroz DM, Vieira CV. Prevalencia del síndrome de burnout entre los anestesistas del Distrito Federal. Rev Bras Anesthesiol. 2015;65(2):104-10. doi: 10.1016/j. bjan.2013.07.016.

24. Soler JK, Yaman H, Esteva M, Dobbs F, Asenova RS, Katić M, et al. Burnout in European family doctors: the EGPRN study. Fam Pract. 2008;25(4):245-65. doi: 10.1093/fampra/cmn038.

25. Mikalauskas A, Širvinskas E, Marchertienė I, Macas A, Samalavičius $\mathrm{R}$, Kinduris $\check{S}$, et al. Burnout among Lithuanian cardiac surgeons and cardiac anesthesiologists. Medicina (Kaunas). 2011;48(9):478-84.

26. Martins AE, Davenport MC, Del Valle MP, Di Lalla S, Domínguez P, Ormando L, et al. Impacto de uma intervenção breve nos níveis de burnout de residentes pediátricos. J pediatr (Rio J). 2011;87(6):493-8. doi:10.2223/ JPED.2127.

27. Dewa CS, Loong D, Bonato S, Thanh NX, Jacobs P. How does burnout affect physician productivity? A systematic literature review. BMC Health Serv Res. 2014;14:325. doi: 10.1186/14726963-14-325.

28. Schaufeli W, Enzmann D. The burnout companion to study and practice: A critical analysis. Boca Raton, FL: CRC press; 1998.

29. Atance Martínez JC. Aspectos epidemiológicos del síndrome de burnout en personal sanitario. Rev Esp Salud Publica. 1997;71(3):293-303.

30. Caballero Martín M, Bermejo Fernández F, Nieto Gómez R, Caballero Martínez F. [Prevalence and factors associated with burnout in a health area]. Aten Primaria. 2001;27(5):313-7.

Correspondencia: Jesus Francisco Maticorena Quevedo

Dirección: Calle Pablo Usandizaga 546, departamento 201. San Borja. Lima, Perú. Teléfono: (+511) 979031880

Correoelectrónico:jesus.maticorena@gmail.com 